\title{
Histoire rurale et Révolution française dans le Japon de la réforme agraire (1926-1953)
}

\section{Robert Calvet}

\section{(2) OpenEdition \\ 1 Journals}

\section{Édition électronique}

URL : https://journals.openedition.org/ahrf/811

DOI : 10.4000/ahrf.811

ISSN : 1952-403X

Éditeur :

Armand Colin, Société des études robespierristes

\section{Édition imprimée}

Date de publication : 1 mars 2003

Pagination : 127-144

ISSN : 0003-4436

\section{Référence électronique}

Robert Calvet, « Histoire rurale et Révolution française dans le Japon de la réforme agraire

(1926-1953) », Annales historiques de la Révolution française [En ligne], 331 | janvier-mars 2003, mis en ligne le 18 avril 2008, consulté le 22 avril 2022. URL : http://journals.openedition.org/ahrf/811 ; DOI : https://doi.org/10.4000/ahrf.811 


\title{
HISTOIRE RURALE ET RÉVOLUTION FRANÇAISE DANS LE JAPON DE LA RÉFORME AGRAIRE (1926-1953)
}

\author{
ROBERT CALVET
}

\begin{abstract}
L'article s'interroge sur le succès précoce des études d'histoire rurale au Japon en liaison avec les structures agraires dominantes et le poids des communautés, et propose l'histoire agraire comme terreau des études révolutionnaires ; l'on relève au passage le rôle de Georges Lefebvre, qui inspira Takahashi Kôhachirô dans ses thèmes de recherche comme dans ses méthodes. L'historiographie japonaise de la question agraire dans le phénomène révolutionnaire jusqu'aux années soixante est marquée par les réflexions sur la rente féodale et sur la réforme agraire de 1946 au Japon. Ces analyses axées sur le passage du féodalisme au capitalisme divergent par ailleurs de celles menées en France, car, plus centrées sur la question des communaux que sur celle de la réclamation de l'autonomie politique par les communautés rurales, elles considèrent le paysan avant tout comme un producteur et non comme un acteur dans le processus révolutionnaire.
\end{abstract}

Mots clés : Japon ; historiographie ; rente féodale ; communautés rurales.

L'historiographie française de la Révolution a longtemps été surtout politique et institutionnelle depuis les recherches menées par les pionniers que furent Aulard et Mathiez. Avant Georges Lefebvre, les grandes villes, et plus encore Paris, accaparaient l'attention des historiens. Les actions menées par les paysans au sein de la Révolution française étaient considérées comme la répercussion des mouvements citadins; seuls quelques travaux pionniers comme ceux de J. Loutchisky (1), P. Sagnac (2) ou A. Aulard (3) avaient abordé la question. En un sens, l'évolution historio-

(1) La petite propriété en France avant la Révolution française et la vente des biens nationaux, 1897.

(2) La législation civile de la Révolution française, la propriété et la famille, 1789-1804, 1898.

(3) La Révolution française et le régime féodal, 1919. 
graphique avait suivi le développement de la science historique au XIXe siècle, et la Révolution française s'était vue appliquer, au fur et à mesure de leur apparition, des problématiques rurales, urbaines, économiques, culturelles.

Au Japon, la fermeture du pays n'avait pas permis de constituer une tradition historiographique (4). Depuis le milieu du XIXe siècle, une réflexion d'abord limitée à l'histoire japonaise s'était élargie à l'histoire de l'Europe et du monde. La Révolution française n'y était pourtant qu'un événement parmi d'autres, dont l'importance ne fut d'abord pas vraiment pressentie. Il est remarquable que l'historiographie japonaise de la Révolution française ait réellement débuté par des recherches spécialisées, axées sur les problèmes agraires, celles menées par Takahashi Kôhachirô (1912-1982) (5), de l'Université de Tôkyô.

On pourrait soutenir que c'est parce que ce pionnier des recherches sur la Révolution a étudié surtout l'histoire rurale que les études révolutionnaires japonaises conservent longtemps une forte coloration rurale. $\mathrm{Ne}$ pourrait-on envisager à l'inverse que c'est parce que les études sur la Révolution française avaient cette forte connotation rurale dès les années quarante que celles-ci ont connu le succès au Japon? Afin de vérifier cette hypothèse, il convient en premier lieu de rechercher les raisons au succès des études d'histoire rurale au Japon, en liaison avec les structures agraires dominantes et le poids des communautés. Pour mieux cerner cette historiographie, il faudra ensuite se demander dans quelle mesure les analyses japonaises de la question agraire dans le phénomène révolutionnaire divergent de celles menées par les chercheurs français, et également entre elles.

L'orientation agraire prise au Japon par les études sur la Révolution résulte d'un ensemble de facteurs. Le premier est évidemment la personnalité même de Takahashi et les relations qu'il entretenait sur le plan international, notamment en France. Très tôt sensibilisé au monde rural, du fait de son lieu de naissance puis de ses premières études universitaires, portant déjà sur les questions agraires dans la Révolution française, Takahashi est rattaché en 1947 au ministère de l'Agriculture qui prépare la réforme agraire. À partir du moment où il enseigne à l'Université de Tôkyô, à l'aube des années cinquante, et où paraît son premier ouvrage sous le titre Shimin kakumei no kôzô [Structure de la révolution bourgeoise], Takahashi prolonge l'étude menée en Europe par

(4) Le tout premier universitaire japonais à s'être intéressé à la Révolution française est Mitsukuri Genpachi (1862-1919), de l'Université de Tôkyô, qui écrivit dès 1919 sous le titre Furansu Daikakumei Shi [Histoire de la grande Révolution française], deux volumes qui ont connu un grand succès public dans l'atmosphère démocratique de l'époque de Taishô, sans parvenir à susciter de vocation universitaire.

(5) En ce qui concerne les noms japonais, nous respectons l'usage de ce pays qui place les noms de famille avant les prénoms, ordre inverse par rapport à la langue française. 
Maurice Dobb (6), et au Japon par Hirano Yoshitarô (1897-1980) et surtout Ôtsuka Hisao (1907-1996) (7).

Takahashi a aussi été fort inspiré par les recherches et les méthodes d'historiens français comme Georges Lefebvre et Marc Bloch, qui, dans respectivement La Révolution française et les paysans et Les Caractères originaux de l'histoire rurale française, accordaient à l'histoire rurale une place prépondérante. Soulignons que l'intérêt de Takahashi pour l'histoire française répond à une curiosité récente mais réciproque de la part des historiens français envers l'histoire japonaise, principalement sa féodalité. Apparue dans la Société féodale (1939-1940) du même Marc Bloch, cet intérêt se retrouve dans les ouvrages de Robert Boutruche (8) et de F. Joüon de Longrais (9), puis dans les bulletins de la Revue historique, à partir de la fin des années cinquante.

Takahashi décide de contacter Lefebvre en 1938, et ce dernier lui envoie un exemplaire de sa thèse sur Les paysans du Nord (...). C'est le début d'une longue relation épistolaire qui amène Takahashi à étudier en France en 19521953, sous la direction d'un Lefebvre qui l'initie personnellement au déchiffrement des archives manuscrites, et lui présente d'autres chercheurs comme Albert Soboul, Richard Cobb ou Georges Rudé. Toutes ces influences ont marqué Takahashi, et l'histoire japonaise de la Révolution en général, puisque son principal contradicteur, Kawano Kenji (1916-1996) (10), a aussi cherché dans le monde rural la raison profonde des événements qui débutent en France en 1789 (11). La même année 1950 où Takahashi publie Shimin kakumei no kôzô [Structure de la révolution bourgeoise], paraît le premier ouvrage de la plume de Kawano, Zettaishugi no kôzô [La structure de l'absolutisme], qui aborde largement les questions agraires, en France et en Angleterre; c'est à travers la doctrine mercantiliste et l'agriculture d'Ancien Régime que Kawano Kenji, économiste de formation, analyse l'absolutisme.

(6) En particulier dans son ouvrage publié à Londres en 1946, Studies in the Development of Capitalism.

(7) Il ne semble pas y avoir eu d'influence réciproque entre Ôtsuka et Dobb, bien qu'ils arrivent à des conclusions analogues sur l'origine du capitalisme, à peu près au même moment. En fait les premiers écrits de Ôtsuka sur cette question sont plus anciens que ceux de Dobb et un peu plus complets. Ces deux chercheurs, Ôtsuka et Dobb, en viennent plus tard tous deux à collaborer avec Takahashi Kôhachirô, puisque Ôtsuka Hisao et Takahashi Kôhachirô codirigent en 1960-1962 l'ouvrage collectif Seiyô keizaishi kôza : hokensei kara shihonshugi e no iko (Discours sur l'histoire économique de l'Occident : la transition du féodalisme au capitalisme); quant à Maurice Dobb, c'est un peu plus tard que Takahashi Kôhachirô réalise avec lui un ouvrage au titre presque identique, Du féodalisme au capitalisme, problèmes de la transition.

(8) En 1947, La crise d'une société porte sur la féodalité japonaise, puis en 1959, Seigneurie et féodalité compare Japon et Occident.

(9) L'Est et l'Ouest : institutions du Japon et de l'Occident comparées, 1958.

(10) Dont nous venons nous-même d'étudier le parcours : Robert CaLVET, Kawano Kenji, le Japon et la Révolution française à la veille du bicentenaire, Thèse pour le doctorat en histoire et civilisations, sous la direction de M. le Professeur Guy Martinière, Université de La Rochelle, décembre 2000.

(11) Et plus généralement le fondement des révolutions, comme en témoignent ses commentaires sur la révolution anglaise de 1642 . 
Comme Takahashi avant lui, pour décrire la crise de la propriété féodale et la réaction qui s'ensuit en France, Kawano s'appuie selon ses propres termes sur «l'analyse détaillée et minutieuse » qu'en donne Marc Bloch dans Les caractères originaux de l'histoire rurale française (1931), ouvrage auquel il empruntait déjà dans Zettaishugi no kôzô et qu'il traduit lui-même en japonais en 1959.

Mais si Takahashi comme Kawano admettent l'importance de l'étude des structures agraires dominantes pour comprendre les phénomènes révolutionnaires, leur interprétation de ces structures et du poids des communautés diverge. Le désaccord découle en partie de la situation des études historiques au Japon, et de la prise de conscience par certains intellectuels de la nécessité d'une réflexion plus profonde sur leur histoire récente et sur l'évolution politique, économique et sociale du Japon depuis la fin du shogunat en 1868 (12). Entre 1927 et 1937, date à laquelle sont arrêtés la plupart de leurs membres, étaient apparues deux écoles de pensée, les écoles Rônô et Kôza. Elles devaient marquer si fortement l'historiographie que l'on continua longtemps à caractériser comme une opposition entre Rônô et Kôza la controverse sur la Révolution française, devenue opposition entre deux universités, Tôkyô et Kyôto, et entre deux chercheurs, Takahashi et Kawano.

Ces deux groupes, essentiellement composés d'intellectuels, voulaient définir le capitalisme japonais; en 1927, puis en 1932 les thèses de la section japonaise du Komintern mettent en avant la nature militariste et "semiféodale » du capitalisme japonais, abandonnant l'interprétation jusqu'alors dominante qui faisait du Meiji ishin une révolution de type bourgeois (13). L'école Kôza découvre le principal soutien de cet état hétérogène chez les propriétaires terriens semi-féodaux (14). Un ouvrage postérieur de 1934 (15) dépeint une société née de Meiji où la paysannerie demeure "semi-serve " et les petits exploitants agricoles «semi-féodaux» sont dépendants des propriétaires, la transformation des marchands en capitalistes ayant produit une société capitaliste retardée. C'est dans une perspective analogue que

(12) Le régime féodal du shógunat cède la place en 1868 à une restauration de l'autorité impériale, dont le pouvoir n'était plus que symbolique et religieux depuis des siècles. En japonais, cet événement prend le nom de Meiji ishin, expression que nous utiliserons de préférence à une traduction française par les mots " restauration » ou « révolution », les événements de 1868 étant complexes, et ne correspondant ni à une restauration monarchique, comme disons celle de Charles II en Angleterre, ni à une véritable révolution.

(13) Comme on pouvait encore le lire dans les écrits de Sakai Toshihiko (1871-1933) ou les premiers textes de Hani Gorô (1901-1983).

(14) En 1932-1933, paraît le Nihon shihonshugi hattatsushi kôza (Discours sur l'histoire du développement du capitalisme japonais) en sept volumes, d'où est tiré le nom du mouvement, le Kóza-ha, littéralement l'école du (dis) cours. Yamada Moritarô (1897-), Hirano Yoshitarô, Noro Eitarô (1900-1934) et Hani Gorô (1901-1983) en sont les principaux membres.

(15) Y. HIRANO, Nihon shihonshugi shakai no kikô : shiteki katei yori no kyâmei (Mécanisme de la société capitaliste au Japon, investigation sur le processus historique). 
Takahashi explique la Révolution française par l'antagonisme entre propriété féodale et capitalisme industriel, ce qui a permis de le rattacher à cette école.

Toujours en 1927, un groupe refusant cette analyse se réunit autour de Yamakawa Hitoshi (1880-1958) et lance le journal Rônô (littéralement «travailleur paysan») (16). Dès 1929, les membres du Rônô-ha publient collectivement Gendai Nihon kenkyû [Études sur le Japon contemporain], et en 1932-1933, Nihon shihonshugi hattatsu shi kôza [Discours sur le développement du capitalisme japonais] (17). En 1937, Sakisaka Itsurô (1897-1985) rédige le Nihon shihonshugi no sho mondai: shihonshugi to nôson no shakaiteki bunka [Ensemble des problèmes du capitalisme japonais, capitalisme et culture sociale des villages], portant le débat sur le rapport entre évolution capitaliste et agriculture. Selon Rônô, la bourgeoisie financière formait déjà avant Meiji des groupes assez puissants pour exercer une réelle hégémonie sur la classe dominante des samurai et des daimyô (18), pour preuve les manufactures dispersées dans les provinces, et l'ascension de commerçants riches à Edo et Ôsaka, devenus prêteurs d'argent; le capitalisme japonais n'était donc pas de nature féodale et le Meiji ishin avait bien été une révolution bourgeoise. Par son intérêt envers les marqueurs de la modernité économique dans le Japon d'avant Meiji, Kawano Kenji se rapproche des thèses de Rônô, alors que Takahashi néglige généralement le rôle du marché. Fondant son analyse sur le régime foncier, Takahashi se désintéresse par ailleurs plutôt des villes et de leur interférence avec le monde rural, oubli qui marque l'historiographie nippone de la Révolution française jusqu'à la diffusion des travaux d'Albert Soboul, dans les années soixante.

La rente féodale, prélevée sur la récolte, était l'un des principaux points de discorde entre Rônô et Kôza et nous retrouvons avec Takahashi et Kawano une querelle commencée avant la guerre. Avant Meiji, le fondement économique du pouvoir des propriétaires consistait en une rente foncière en nature. Pourtant, du fait de la fréquence de l'activité salariée, les fermiers dépendaient du marché; même la terre était devenue une marchandise s'échangeant librement. Kushida Tamizô (1885-1934), de Rônô, avait soutenu (19) qu'à cause de la propriété privée du sol et de la libre circulation de main-d'œuvre, on ne pouvait considérer la rente foncière comme simplement féodale, mais que, presque monétisée, elle était

(16) Le sigle choisi pour la revue socialiste Rônô, formé à partir des idéogrammes rô (de rôdôsha, travailleur) et nô (de nômin, paysan) témoignait du souci de s'appuyer sur la paysannerie autant que sur le prolétariat, contrairement au choix qui se fit en Occident, où le monde rural était volontiers associé aux idéologies conservatrices.

(17) Parmi les plus importants des membres de Rônô-ha, citons Ôchi Hyôe, futur président de l'Université Hosei.

(18) Les samurai sont des guerriers, les daimyô des seigneurs détenant un fief.

(19) Dans Nôgyô mondai (Problèmes de l'agriculture), Kushida Tamizô Zenshû, t. 3, 1935. 
pré-capitaliste. Les membres du Kôza-ha, Yamada et Hirano, avaient répliqué que des secteurs d'économie de marché pouvaient parfaitement se développer au sein de rapports de production féodaux. Selon Hirano, l'examen des rapports de production dans l'agriculture prouvait que le propriétaire en détenait le contrôle effectif, et les pratiques coutumières exerçaient un pouvoir coercitif non économique sur les rapports de production; il en concluait à la nature semi-féodale de la rente.

La survivance de pratiques féodales reste difficile à prouver ou à infirmer dans le cas des structures sociales particulières propres à la riziculture inondée à haut rendement; c'est pourquoi les chercheurs de la tendance Kôza se sont attachés à certains districts montagneux du Japon, où les survivances féodales dans l'agriculture étaient indiscutables, et où l'on vit même exceptionnellement une forme de servage se perpétuer jusque dans les années 1920, au moment où la controverse entre Kôza et Rônô bat son plein. En revanche, les membres du Rônô-ha insistaient sur la latitude décisionnelle du fermier dans le choix des assolements, l'utilisation d'engrais, l'achat d'instruments, l'irrigation. Considérant trop fragmentaires et surtout sans lien direct avec les rapports de production les indices de pratiques coutumières avancés par Kôza , ils concluaient à la disparition du servage et au remplacement des corvées par une rente, même dans les villages les plus reculés du pays, qualifiés par Kôza de "typiquement féodaux» (20). En fait, Kôza et Rônô tombaient d'accord sur l'existence de rapports de production à la fois «féodaux » et «capitalistes », en divergeant sur leur part respective. Presque exclusivement attachée à la situation agraire, et plus encore à son aspect législatif, l'école de Kôza ne prenait pas toujours en compte les évolutions concrètes du monde rural.

Dans l'analyse de Takahashi, la rente reste caractéristique du féodalisme, de même que les restrictions imposées par le shôgunat aux cultures industrielles ou le statut juridique du paysan japonais, proche du servage. En fin de compte, fidèle à la conception de Kôza qui interprétait les fermages comme une dìme entretenant la misère paysanne, Takahashi pouvait affirmer que le Meiji ishin, loin d'avoir aboli le parasitisme des propriétaires, l'avait au contraire renforcé et légalisé. Kawano, sur la foi d'exemples dans le village de Wakae ou la région d'Owari près de Nagoya, constate l'apparition dès la deuxième moitié du XVIII siècle de «paysans-

(20) TsuCHIYA (Takao), Edo jidai no keizai (L'économie à l'époque Edo) et Kinsei Nihon nôson keizai shiron (Essai historique et économique sur les communautés rurales du Japon moderne), 1933. Pourtant chacune des factions connaissait ses divergences. Inomata Tsunao ou Kuchida Tamizô de Rônô expliquent la coexistence au Japon d'un capitalisme dominant dans l'industrie et d'une société rurale apparemment semi-féodale. Inomata admet que l'agriculture soit restée en proie à des rapports de production semi-féodaux, à la différence de presque tous les membres du Rônô-ha, mais l'explique par le développement de l'impérialisme plus que comme une survivance féodale à proprement parler. Quant à Yamada, le chef de file de Kôza-ha, il allait au-delà d'une simple réflexion sur l'agriculture, reliant les problèmes du monde rural à ceux de l'industrie. 
fabricants » employant des ouvriers et ayant abandonné le travail agricole pour un travail manufacturier; il en déduit une différenciation de la paysannerie dans un sens capitaliste (21). Pour Takahashi le fait majeur est l'appauvrissement et l'endettement général de la paysannerie, finalement réduite à un ensemble de petits fermiers dépendants des grands propriétaires absentéistes, alors que Kawano note l'opposition croissante entre une classe de paysans riches employant une domesticité et des paysans pauvres ne possédant pas même un hectare, réduits à louer leur travail sur la terre d'autrui. Takahashi et Kawano se montrent plus nuancés que leurs prédécesseurs; Takahashi note ainsi que la paysannerie japonaise s'est déjà largement engagée dans la différenciation dès la fin du XVIIe siècle.

L'influence sur Takahashi d'historiens du début du siècle spécialisés dans l'histoire rurale, surtout celle de l'époque d'Edo, est enfin à relever (22). Au premier rang de ces chercheurs, signalons un membre du Kôza-ha, attaché depuis les années trente à l'étude du Japon rural et internationalement reconnu, Furushima Toshio (1912-1995) (23). Son analyse des structures sociales et communautaires situe la ruralité japonaise dans un cadre nettement féodal, en choisissant ses exemples dans les régions les plus isolées du Japon (24), mais il ne néglige pas le développement de l'agriculture commerciale sous les Tokugawa (25).

Lhistoire rurale connaissait un fort développement au Japon avant Takahashi, avec les travaux de Nakamura Kôya (1885-1970) sur l'époque Tokugawa (26) ou d'Aruga Kizaemon (1897-1979) sur le servage et le fermage (27). De plus, historiens et économistes montrent un vif intérêt

(21) Au point que sur la fin de la période Tokugawa, des cas se rencontrent où près de la moitié de la population d'un village est engagée dans l'industrie textile, "fait remarquable dans une société féodale". Kenji KaWANO, "Révolution française et révolution de Meiji. Aspects économiques et sociaux ", Annales historiques de la Révolution française, 1987, p. 10.

(22) Déjà en 1905, l’ouvrage précurseur de Kawakami HAJIME (1878-1946), le Nihon sonnô ron (Arguments pour l'agriculture japonaise), plaidait comme les physiocrates français pour accorder à l'agriculture une place centrale dans l'économie par rapport au commerce considéré comme périphérique.

(23) Maintes fois réédité, son ouvrage le plus impressionnant reste Kinsei Nihon nôgyó no kôzô (La structure de l'agriculture japonaise à l'époque «pré-moderne ", 1943), où «époque pré-moderne » traduit le shôgunat. Furushima contribue plus récemment au volume 4 de la prestigieuse Histoire du Japon sous la direction de J. W. Hall de l'Université de Cambridge, grâce à l'article intitulé « The Village and Agriculture during the Edo Period ".

(24) Hôken jidai kôki no nômin no seikatsu (La vie des paysans à la fin de l'époque féodale, 1948) Nihon hôken nôgyô shi - kazoku keitai to nôgyô no hattatsu (Histoire de l'agriculture féodale japonaise : structures familiales et développement de l'agriculture, 1941) - Nihon rin'ya seido no kenkyû : kyôdô teki rin'ya shoyû o chûshin ni (Études sur le système du village forestier : au cour de la propriété communautaire dans le village forestier, 1955).

(25) Kinsei shôgyôteki nôgyô no tenkai (Le développement de l'agriculture commerciale à l'époque «pré-moderne »), 1950. 1949).

(26) Kinsei nôson shakai shiron (Essai historique sur les sociétés rurales à l'époque "pré-moderne ",

(27) En particulier Daikazoku seido to nago seido - nanbu Ninohe-gun Ishigami-mura ni okenu (Système de la famille élargie et système de servage dans le village de Ishigami, région méridionale district de Ninohe, 1939) et Nihon kazoku seido to kosaku seido (Système de la famille et système de fermage au Japon, 1943). 
pour l'histoire rurale européenne, surtout anglaise, dans un souci de comparatisme. Toya Toshuki publie en 1951 une étude sur la yeomanry anglaise, Horie Hideichi traduit en 1942 l'ouvrage de Thomas Mun $A$ discourse of trade, from England unto the East-Indies (28), et c'est encore l'agriculture anglaise qui intéresse l'historien Kurihara Hakuju ou l'économiste Tobata Seiichi. Takahashi reste donc le premier à réfléchir en comparaison avec le cas français, et plus précisément la période révolutionnaire.

L'histoire du XXe siècle et des premières révolutions socialistes avait ouvert les yeux des historiens sur la place des problèmes agraires dans le déclenchement et le déroulement des révolutions. Comment ignorer la question agraire dans l'origine ou l'évolution de la Révolution alors que c'est dans des pays majoritairement ruraux, comme la France de 1789, le Japon de 1868 , ou aussi bien la Russie du XXe siècle que se produisent les révolutions? Les mêmes questions se posent en théorie dans tous ces cas. Replacer la question agraire au cœur de la révolution, c'est mener une réflexion plus large sur les révolutions du $\mathrm{XX}^{\mathrm{e}}$ siècle, et chercher en même temps à expliquer une des contradictions les plus embarrassantes entre la théorie marxiste et la réalité historique, comment la révolution prolétarienne prévue par Marx s'est réalisée dans les pays les moins industrialisés, où dominait la paysannerie. Bien que la classe paysanne n'ait d'abord pas été perçue comme moteur des révolutions par ceux qui en furent les principaux acteurs, comment être insensible à l'évolution de l'analyse du rôle des paysans, entre la révolution russe et la révolution chinoise? Le Japon de l'après-guerre, avec sous les yeux le récent exemple de la Chine, pouvait se montrer plus sensible à une théorie révolutionnaire faisant une large place à sa composante paysanne.

Mais les années où débute la controverse entre Takahashi et Kawano sont surtout celles où s'est mise en place la réforme agraire japonaise de 1946, puis son application. Le problème de la redistribution du sol dans un pays où la terre cultivable est si disputée a généré une réflexion sur l'histoire agraire et sur les modes de propriété du sol dominants dans le passé. Sur le simple plan matériel, la réforme est essentielle à la naissance des études d'histoire rurale, car les données sur l'histoire agraire jusque-là disséminées dans des fonds d'archives privés ont été centralisées dans les toutes nouvelles Archives nationales. Des études étaient effectuées à l'échelle du pays entier par les nombreux bureaux et commissions rendus nécessaires par l'application de la réforme agraire; nous avons pu constater que Takahashi le premier y avait participé.

Il existe bien tout un faisceau de causes ayant permis l'essor des études d'histoire rurale au Japon et l'application de cette spécialité en particulier à

(28) Les doctrines exposées dans cet ouvrage font de Thomas Mun (1571-1641) l'un des premiers mercantilistes. 
l'histoire de la Révolution française. Orientation particulière de cette historiographie, le comparatisme rend difficile un traitement séparé de la réflexion menée sur la Révolution française et de celle conduite sur le Meiji ishin, puis sur la réforme agraire japonaise de 1946. Notre analyse commencera donc par s'attacher aux divergences de vues entre une conception française avec laquelle nous sommes plus familiers et l'analyse menée au Japon, avant de s'intéresser aux différences entre chercheurs japonais, en relation avec l'histoire rurale française et européenne.

À trop souligner que les études menées par Takahashi et Lefebvre sont centrées sur la question agraire dans la Révolution, on oublierait que leur argumentation est différente. Chez Lefebvre, le monde rural était un agent et non des moindres de la Révolution, la Grande Peur qui enflamme les campagnes ayant pesé sur « l'abolition du régime féodal » pendant la nuit du 4 août. Il pouvait cependant aussi être un facteur d'opposition à une révolution trop radicale. Mais la révolution paysanne ne reste chez Lefebvre qu'une composante au sein d'une Révolution essentiellement bourgeoise, alors que pour Takahashi ce sont les évolutions du monde rural qui sont au cœur du processus révolutionnaire, et même le soutiennent. Là où la réforme agraire réclamée par les campagnes françaises est perçue comme un retour en arrière par un renforcement de la solidarité paysanne face aux contraintes d'un marché naissant, celle que demandent les campagnes japonaises est plutôt anti-féodale et en devient, même sans l'avoir désiré, procapitaliste.

Takahashi explique que l'histoire agraire en est venue à constituer pour lui le nœud du problème, parce que la « féodalité [est] non seulement une institution sociale, mais aussi [...] un mode historique de production, [...] fondé, au Japon comme en Europe occidentale, sur la propriété foncière [...], forme élémentaire de la richesse sociale dans la société précapitaliste ". Considérée comme la «base de la société féodale », la communauté rurale avec « les droits seigneuriaux et rentes féodales et les contraintes communautaires » qui lui sont liés, doit disparaître pour permettre «la transition de la société féodale à la société capitaliste moderne", "tâche principale de la révolution bourgeoise » (29). Le concept de révolution paysanne développé par Takahashi est centré sur la seule recherche de l'abolition totale du féodalisme, alors que Lefebvre considérait la révolution paysanne comme un mouvement à la fois anti-féodal et anti-capitaliste. Le chercheur japonais considère que la paysannerie n'existe qu'aussi longtemps que se maintient le féodalisme, cédant ensuite la place à une bourgeoisie et un prolétariat ruraux, ce que Lefebvre remarquait aussi, mais l'étroitesse du lien établi par Takahashi entre paysannerie et féodalisme explique certaines de ses omissions. 
Concernant le rôle des campagnes dans la Révolution française, il y a une différence fondamentale de démarche chez Georges Lefebvre; Takahashi Kôhachirô ou Kawano Kenji. Chez Lefebvre, le souci est méthodologique, il s'agit d'approcher sous un angle différent, par l'étude de sources peu mises à contribution jusque-là, une Révolution française déjà bien connue, et de dégager les éléments d'autonomie de la révolution paysanne. Chez Takahashi, la question est plus philosophique ou politique: sa théorie de la transition du féodalisme au capitalisme se développe à partir de l'analyse comparée entre Japon et Europe des structures sociales en milieu rural. Ayant remarqué, par exemple sur l'exemple espagnol ou néerlandais, que le développement du commerce extérieur et l'ascension sociale des négociants n'aboutissaient pas forcément à la formation du capitalisme industriel, c'est dans l'industrie rurale et son opposition aux corporations urbaines que, de même que Dobb, Takahashi trouve l'origine de l'industrie moderne (30), et le modèle universel de l'évolution des sociétés vers la modernité. Pour lui, « la catégorie sociale qui met fin au féodalisme, [...] doit être cherchée parmi la classe des moyens et petits producteurs entrepreneurs et des petits producteurs de services (la petite bourgeoisie), plutôt que chez [...] la haute bourgeoisie, qui reste attachée à la monarchie absolue » (31).

Georges Lefebvre dans "La Révolution française et les paysans", publié en 1933 dans les Annales historiques de la Révolution française, partait de la conviction que la culture paysanne était précapitaliste. Lorsqu'il écrit qu'il y a «une révolution paysanne, qui possède une autonomie propre quant à son origine, à ses procédés, à ses crises et à ses tendances ", il signifie que, au sein d'une révolution bourgeoise et capitaliste, les paysans s'ils ne cherchaient pas toujours à renouer avec ce passé précapitaliste, désiraient en tout cas protéger les institutions paysannes précapitalistes de l'économie de marché, qui «aggravait leurs conditions d'existence» (32). Les droits communaux sont le point nodal du caractère précapitaliste de la paysannerie pauvre : «Toutes les pensées du paysan pauvre tendaient donc à limiter le droit de propriété individuelle pour défendre les usages collectifs, qui lui permettaient de vivre et qu'il regardait comme une propriété aussi sacrée que les autres, et pour empêcher que les denrées alimentaires nécessaires à son existence lui devinssent inaccessibles " (33). Mais l'économie à laquelle

(30) Cette théorie apparaît dans «Du féodalisme au capitalisme : problèmes de la transition », Science and Society, vol XVI, $\mathrm{n}^{\circ} 4,1952$, pp. 35-36, et est développée largement dans le Kindai shakai seiritsu shiron (Essai historique sur l'établissement de la société moderne, 1947), aux pages 1 à 22 qui s'essaient à une typologie de la structure du capitalisme moderne, et aux pages 189 à 226 présentées comme une « introduction à l'étude de la Révolution française».

(31) Kindaika no hikakushiteki kenkyû (Études comparatives d'histoire de la modernisation), pp. 117-118.

(32) Georges Lefebvre, "La Révolution française et les paysans », p. 250.

(33) Id., p. 253. 
Georges Lefebvre se réfère est un mélange de terres privées et collectives propre surtout à la France rurale du Nord, où chaque famille possédait des bandes de terre arables non encloses au sein de vastes champs, la décision de ce qui y serait cultivé étant prise collectivement par le village. Sur les terres en jachère, friches ou chaumes des champs déjà moissonnés, l'usage du droit de pâture permettait aux paysans les plus pauvres d'élever un animal et de disposer d'un revenu minimal nécessaire à leur-survie. Léthique paysanne décrite par Lefebvre reposait sur une morale de subsistance, pertinente dans les lieux étudiés, mais à utiliser avec précautions dans d'autres provinces françaises déjà perturbées par l'intrusion de l'industrie (34), qui fournissait à nombre de familles un revenu d'appoint.

Au Japon, des zones « modernes » où prédomine la riziculture inondée ou des cultures industrielles, coexistent avec des zones montagneuses peu productives aux structures archaïques. La riziculture impose d'enclore les champs afin de les inonder, donc des contraintes collectives, mais la culture en elle-même reste aux mains de l'exploitant et de sa famille, qu'il soit propriétaire ou locataire. Takahashi en déduisait que la riziculture a déve-

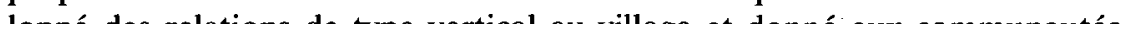


paysannes commencent à être assez organisées pour résister, et souvent victorieusement, à la pression fiscale. Les révoltes paysannes assorties de violence ne cessent pas sous les Tokugawa, puisque Aoki Kôji en recense environ trois mille sur deux siècles et demi, soit en moyenne une par mois (36). Sur la fin du shôgunat, elles deviennent plus nombreuses, jusqu'à leur apogée en 1866 avec les soulèvements de Fukushima, Shinobu et Date, puis de l'année suivante dans la région de Nagoya. Dans les années 1873-1877, c'est contre la réforme de l'impôt foncier de 1873 que tout le pays, et surtout le Kyûshû, se soulève avec une vigueur qui n'a rien à envier aux jacqueries armées des années 1789-1793 en France; l'impact en est si fort que le gouvernement japonais procède à une baisse de l'impôt. Si le rôle des communautés rurales dans l'organisation des révoltes s'efface progressivement devant celui des individus, ce n'est pas avant le début de l'ère Meiji.

Il peut paraître curieux qu'avec sous les yeux de tels exemples, aucun chercheur japonais n'ait mis l'accent, comme le fit F. Gauthier en France, sur la réclamation de l'autonomie politique par les communautés rurales, pourtant perceptibles dès 1866 dans le récit anonyme des émeutes (37). Mais fidèle à Georges Lefebvre, Takahashi réfléchit surtout aux modalités de passage à la propriété privée des terres communautaires. Pourtant, alors que les communaux étaient au centre de l'évolution des campagnes françaises, au Japon les seules terres qui passent en partie des communautés à l'État sont les forêts. L'élevage n'ayant jamais eu la même importance qu'en Europe, même si les légumes cultivés en montagne et l'appoint de gibier n'étaient pas négligeables dans l'alimentation, ce sont plutôt la pêche et la culture de pousses comme le soja, effectuée sur la parcelle même, qui procurent les protéines. Enfin, la situation du paysan japonais avant Meiji était plus complexe que celle du paysan français sous l'Ancien Régime, l'exiguïté des parcelles ayant souvent poussé un propriétaire à louer une terre en sus de celle lui appartenant en propre, voire à offrir son travail à un autre propriétaire. Sans nier la pauvreté réelle du paysan japonais, caractériser ses motivations par la seule éthique de la subsistance est plus difficile.

La controverse entre Takahashi et Kawano est d'une nature différente de la divergence de vues entre Lefebvre et Takahashi. Il s'agit moins de définir la place de la révolution agraire dans la Révolution que de comprendre la position des sociétés agraires au sein d'un schéma marxiste d'évolution des sociétés, du féodalisme au capitalisme, mais avec moins de variété qu'Ado concernant les voies dans cette marche vers le capitalisme. Il est pratique de partir de l'analyse proposée par Henri Sée dans Les origines $d u$

(36) Kôji AOKı, Hyakushô ikki no nenjiteki kenkyû (Étude chronologique des révoltes paysannes), Tôkyô, Shinseisha, 1966.

(37) Ôshû Shinobu-gun Date-gun no ohyakushô-shû ikki no shidai (Circonstances du soulèvement paysan dans les districts de Shinobu et de Date, dans l'Ôshû). 
capitalisme moderne, parce qu'elle a connu au Japon un certain succès (38). Sée distinguait deux types de régions industrielles : dans le premier, des «villages pauvres aux sols épuisés » et une quasi-absence de développement urbain expliquent le manque de concurrence entre industrie rurale et urbaine, comme en Bretagne ou dans le Bas-Maine. Toutes les étapes de la fabrication de toiles, depuis la production du chanvre jusqu'à la transformation en produit fini, sont assurées par les paysans, les marchands ne participant guère au processus. Dans le deuxième type défini par Sée au contraire, régions prospères et à l'industrie urbaine vigoureuse comme la Flandre, la Picardie ou la Haute-Normandie, l'industrie rurale mène à l'industrie capitaliste par la transformation du négociant-entrepreneur en patron industriel (39).

Cette interprétation de Sée avait déjà été critiquée par Ôtsuka Hisao dans sa Généalogie du capitalisme moderne [Kindai shihon shugi no keifu], notamment sur la domination des campagnes par les capitaux marchands urbains comme expression de la poussée du capitalisme moderne; au contraire, Ôtsuka oppose capital industriel et capital marchand (40). Dans sa lignée, Takahashi comme Kawano continuent dans les années cinquante à voir « la base originelle du développement bourgeois » dans l'indépendance que l'industrie rurale acquiert par rapport aux villes (41). Mais leur interprétation diverge rapidement. Takahashi résume la révolution bourgeoise au « développement des manufactures, c'est-à-dire [à] l'accès au pouvoir du capital industriel ». Kawano trouve cette explication trop simpliste, et juge impossible de condenser les origines de la Révolution aux «exigences de deux fractions d'une même bourgeoisie, qu'elle soit marchande ou industrielle ». Plus largement, il refuse de limiter le déclenchement de la révolution bourgeoise au seul jeu des forces économiques, et propose de le " comprendre comme l'expression d'un ensemble de forces de nature plutôt politique ou en tout cas extérieures à l'économie ", reposant d'une part sur « les relations conflictuelles liées à la condition sociale et aux privilèges », et d'autre part «sur une opposition de régime politique et de vues dans la manière de structurer la société » (42). Kawano nie que la Révolution française ait permis le progrès de l'industrialisation, et critique Ôtsuka pour qui la révolution bourgeoise amena la suprématie du capitalisme industriel. Une relation de cause à effet lui semble loin d'être établie, l'accumulation

(38) Les origines du capitalisme modeme (1926) est traduit en japonais dès 1936 par Ôbuchi Shôzô (1899), et connaît même une nouvelle traduction en 1954, par Tsuchiya Sôtarô et Izumi Shizuo. Quant à Matérialisme historique et interprétation économique de l'histoire (1927), il avait été traduit dès 1931 par Hanazawa Takeo, et reste aujourd'hui l'ouvrage de Sée le plus diffusé au Japon.

(39) Henri SÉE, Les origines du capitalisme moderne, pp. 136-137.

(40) En Europe, Maurice Dobb trouvait la genèse du capitalisme industriel dans l'auto-dissociation du mode de production paysan, les petits producteurs se libérant de la propriété foncière.

(41) Zettai shugi no kôzô (La structure de l'absolutisme), 1950, pp. 47-48.

(42) Id., p. 55. 
primitive consolidant le capital industriel ayant progressé de manière significative seulement après la Révolution.

Outre la question déjà évoquée de la rente féodale, la contradiction la plus vive entre Takahashi et Kawano porte sur la suppression des droits féodaux et la vente des biens nationaux en France. Takahashi cite une remarque de Lefebvre : «La réforme agraire japonaise qui suivit la Seconde Guerre mondiale a été plus radicale que celle de la Révolution française ", qu'il explicite ainsi : le système de propriété féodal en France a été supprimé sans indemnité, contrairement à ce qui s'est produit dans le Japon de Meiji. Alors que la Révolution avait réussi à affranchir totalement les paysans des charges seigneuriales, Meiji avait été incapable de créer des paysans indépendants et libres, preuve de ce caractère incomplet. Ensuite, alors que les paysans français pouvaient élargir leur propriété par l'achat de biens nationaux, les paysans japonais étaient au contraire dépossédés de leurs terres communautaires par l'État.

Takahashi juge que ces deux évolutions sont caractéristiques de deux systèmes antagonistes, le système occidental dans lequel le producteur devient commerçant, et le système oriental où le marchand devient fabricant. En Europe, l'évolution permet l'apparition de paysans libres et indépendants relevée plus tard par Ado, et considérés de même que chez Dobb comme la base du développement capitaliste. En Asie, ni ces paysans libres ni la classe moyenne bourgeoise n'étant autorisés à se développer, la société démocratique ne peut naître. Takahashi n'oublie ni la longueur ni la difficulté de la disparition du régime foncier féodal en France, des décrets du 11 août 1789 jusqu'à la décision entre juin et août 1792, puis en juillet 1793, de supprimer définitivement les droits. Il distingue de ce fait à partir de l'évolution de la propriété foncière deux étapes dans la Révolution : favorable aux seigneurs, la première voit la simple régularisation des charges féodales et leur remplacement par un paiement en argent, et n'est pas véritablement révolutionnaire; mais la seconde, lors des années 1792-1793 fait disparaître les résidus féodaux (43). Cette interprétation permettait à Takahashi en 1950 de faire des Girondins les partisans du féodalisme et des Montagnards ceux du capitalisme, explication qui hérissa Albert Soboul (44).

Kawano Kenji souligne plutôt les à-coups du processus, en rapprochant le rachat des droits féodaux de l'accès futur à la propriété foncière pour les petits paysans. Il note que si l'abolition du régime féodal s'était décidée en six heures seulement, six jours avaient été nécessaires pour la codifier,

(43) Shimin kakumei no kôzô (La structure de la révolution bourgeoise), 1950. Selon lui, le Japon de Meiji était resté bloqué entre les deux étapes.

(44) Par la suite, Takahashi rectifia sa position pour considérer Girondins et Montagnards comme tous deux «bourgeois». Kindaika no hikakushiteki kenkyâ (Études comparatives d'histoire de la modernisation), ouvrage posthume publié en 1982. 
pendant lesquels grands propriétaires et privilégiés avaient vidé la décision initiale de sa substance. Pour lui, l'essentiel dans les décrets du 11 août est que le mode d'abolition étant le rachat, il n'y a pas eu d'abolition à proprement parler (45). Ainsi, il estime que la domination de classe sur la paysannerie a été précipitée, en autorisant une grande partie des paysans riches à entrer dans la peau de propriétaires fonciers, et en permettant la transformation de la propriété féodale en propriété moderne. Cette modernisation est conçue comme un embourgeoisement des campagnes, les paysans ne pouvant s'acquitter des indemnités liées à l'abolition devenant de simples ouvriers agricoles (46).

Selon Kawano, le passage à la propriété foncière moderne est surtout symbolisé par les vicissitudes touchant la vente des biens du Clergé. À l'origine, les décrets du 14 mai 1790 prônaient la vente de petits lots avec paiement échelonné sur douze ans, ce qui laissait aux paysans pauvres un petit espoir de devenir propriétaires. Kawano explique que les difficultés financières ont vite conduit à abandonner cette politique. Le 3 novembre 1790, le principe de ne plus morceler la terre et d'un paiement par avance sur quatre semestres met un terme au rachat par les paysans pauvres; il aboutit à un accaparement sous forme de grands domaines par "entre autres des citadins, des fournisseurs désireux de spéculer et des paysans riches », ce qui permet à Kawano d'affirmer que «là encore s'accomplissait le principe d'une révolution par le haut " (47).

Kawano rappelle que c'est seulement sous la pression des Hébertistes qu'est décidé le 13 septembre 1793 un système de vente favorisant les paysans non propriétaires, mais insiste sur le choix par les gouvernements révolutionnaires du principe des enchères, et en particulier sur le décret du 24 avril 1793 interdisant aux «associations des habitants d'une commune » l'achat d'une terre en commun (48). Kawano en conclut que la paysannerie dans son ensemble n'a guère profité de la vente des biens nationaux, et que les décrets n'ont pas réussi à transformer les paysans en propriétaires fonciers, ce que Lefebvre notait déjà (49).

Ce sont encore les conclusions de Takahashi que Kawano met en doute, mais le problème posé n'est pas le même. Takahashi veut prouver l'existence de la transition bourgeoise dans le mode de propriété de la terre. Ce qui intéresse Kawano c'est de savoir qui s'est emparé des biens nationaux, c'est-à-dire de juger la Révolution dans ce qu'elle a apporté au peuple. La divergence de vues tourne autour des modalités de la transition entre deux

(45) Shimin kakumei ron (Traité sur la révolution bourgeoise), 1956, p. 16.

(46) Id., pp. 16-17.

(47) Id., p. 18.

(48) Articles 21, 22 et 23.

(49) Shimin kakumei ron, p. 63. 
modèles économiques, du féodalisme au capitalisme. Transition plutôt brusque ou graduée, et surtout moment de cette transition, voilà le cœur du problème. Takahashi cherche à éloigner le cas japonais du cas français, mettant en évidence les divergences et les aspects rétrogrades de la réforme de l'impôt foncier sous Meiji, alors que Kawano au contraire rapproche l'évolution des deux pays. Une démarche possible à condition pour Takahashi de faire de la Révolution française le modèle classique de révolution bourgeoise, et de Meiji une révolution incomplète ayant créé une société semi-féodale (50). En revanche Kawano, considérant Meiji comme une révolution bourgeoise à part entière même si elle fut moins avancée que la Révolution française, se devait de présenter cette dernière comme complexe plutôt que comme modèle. C'est possible à condition de peindre une féodalité en état de décomposition déjà avancé avant 1789 en France et avant 1868 au Japon, et de montrer que dans un cas comme dans l'autre ces événements n'amènent pas directement la suprématie du capitalisme.

Malgré cette optique très différente, le schéma suivi par les deux hommes s'articule autour d'un événementiel scandé par des dates symboliques des ruptures. Le partage et la revente des biens nationaux en France, et au Japon le chiso kaisei puis le nôchi kaikaku, les deux réformes, celle de Meiji et celle de 1946. Les deux chercheurs ne discutent pas la validité du passage d'un type de société féodal à une société bourgeoise, ce qui distingue nettement Kawano de Furet. Le développement de l'État moderne, et dans la Révolution française la période de la dictature jacobine puis le dépérissement de la dynamique révolutionnaire en institution impériale, sont devenus plus tard une problématique de Kawano. Pourtant, chez Kawano comme chez Takahashi, le monde rural disparaît de l'analyse quand elle devient plus politique, et les évolutions du monde paysan restent confinées aux modes de propriété du sol. La politisation précoce des comportements paysans dès l'Ancien Régime, dont l'étude fut initiée par R. Dupuy et par J.-P. Jessenne, échappe totalement aux chercheurs japonais de la génération de Takahashi ou de Kawano, qui considèrent les paysans comme des producteurs, jamais comme les porteurs éventuels de nouvelles valeurs démocratiques. N'oublions pas que ces perspectives sur l'histoire rurale en France ont été permises par la publication de monographies locales, comme l'étude de l'Artois par Jean-Pierre Jessenne, de la Champagne par Claudine Wolikow, ou de l'Oise par Guy-Robert Ikni,

(50) C'est à Hirano que l'on doit d'avoir qualifié le premier la Révolution française de révolution bourgeoise classique, une interprétation qui connaît au Japon une extraordinaire pérennité. Mais cette analyse est aussi faite dans le but d'expliquer Meiji ishin comme une révolution bourgeoise incomplète. Dans les écrits de Hirano, le capitalisme est considéré sous l'angle de son développement global, ce qui donne une conception très téléologique de l'histoire; ceci s'explique par l'impossibilité d'accéder aux sources de l'histoire occidentale, qui oblige Hirano à développer sa réflexion exclusivement à partir des écrits de Marx et de Engels. Yoshitarô HiRaNo, Nihon shihonshugi shakai no kikô: shiteki katei yori no kyûmei, Tôkyô, Iwanami shoten, 1934. 
travaux hors de portée pour les chercheurs japonais d'alors. Mais plus largement, comment se lancer dans de telles études tant que n'avait pas été posé puis dépassé le problème de la confrontation des schémas marxiste et révisionniste, et recherchées de nouvelles définitions de catégories sociales dans la société paysanne allant au-delà de la simple étude de cas, ce qui ne se fait pas en France avant les années 1980 ?

Parce qu'elle privilégiait l'agriculture «féodale » des Tokugawa et celle «bourgeoise » mise en place avec l'occupation américaine, l'analyse japonaise de l'évolution agraire du pays a laissé dans l'ombre ce qui se produisit entre la mise en place du chiso kaisei et celle du nôchi kaikaku. Ce que les chercheurs japonais ont surtout négligé dans leur modèle, et qui avait indiscutablement échappé aux historiens de l'après-guerre, obnubilés par le problème général de la transition du féodalisme au capitalisme et son application au cas japonais, c'est l'impact de la période Taishô et de la montée du militarisme sur les sociétés agricoles d'avant la réforme agraire de 1946.

Le passage sous Meiji à un lourd impôt en argent avait endetté beaucoup de petits exploitants, qui avaient ensuite perdu leur parcelle, d'où une concentration des terres. Mais le gouvernement japonais avait vite réalisé la menace d'une agitation paysanne, et s'il s'était efforcé d'éradiquer l'influence grandissante des syndicats et des partis de gauche, il avait aussi envoyé des médiateurs locaux, les kosakukan, bien obligés d'affronter les propriétaires. Durant la montée du totalitarisme japonais, l'influence locale des propriétaires fonciers est de moins en moins acceptée par le pouvoir central. De plus, des conditions économiques désavantageuses et la raréfaction de la main-d'œuvre agricole provoquent un affaissement de la production, encore aggravé par la sécheresse de 1939. Le gouvernement japonais accroît son emprise sur le marché des denrées de première nécessité et il en résulte paradoxalement une amélioration de la situation des fermiers et une restriction des droits des propriétaires. Entre 1939 et 1941, les loyers et les prix d'achat sur la terre, sont gelés voire diminués dans le but de décourager son passage aux mains des plus grands propriétaires. La production de riz est particulièrement contrôlée avec un prix d'achat garanti en 1942, multiplié par sept de 1942 à 1946, ce qui fait croître exponentiellement le revenu des fermiers, alors que celui des propriétaires stagne. Soucieux d'assurer la subsistance de tous pour maintenir l'ordre et se maintenir, les militaires avaient ouvert la voie à la perte d'influence des propriétaires et à la transformation des fermiers en propriétaires exploitants. Ce contrôle des prix par l'État, qui rappelle celui mis en œuvre lors de l'épisode jacobin dans une période elle aussi marquée par l'économie de guerre, n'a certainement pas été étranger au succès de la réforme de 1946, et d'une agriculture plus individualiste, recentrée sur les petits propriétaires. 
Nous pouvons déduire de ce rapide examen des études historiques au lendemain de la Seconde Guerre mondiale sur deux problèmes particuliers, l'histoire rurale et la Révolution française, que les influences ont été réciproques. Chez les chercheurs qui se sont attachés à la fois à ces deux domaines, ce qui fut la règle plus que l'exception, la façon de percevoir l'évolution de leur propre pays a influencé l'interprétation de la Révolution française tout autant que l'étude de cette révolution modifiait leur vision de l'histoire du Japon. Le fait d'avoir considéré la Révolution française comme l'exemple type de la révolution bourgeoise a engagé l'analyse de Meiji dans certaines voies, ce qui était déjà assez connu en France où la controverse entre Rônô et Kôza n'était pas ignorée. Mais l'étude des conditions agraires et des structures sociales japonaises ont tout autant tiré l'interprétation de la Révolution française dans certaines directions. Pour des chercheurs comme Takahashi Kôhachirô qui avaient été convaincus par leur analyse de l'aspect féodal du monde rural japonais avant Meiji, les petits exploitants fermiers dans la Révolution française cherchaient à se débarrasser du féodalisme. Pour d'autres qui comme Kawano Kenji, s'étaient attachés à l'étude des doctrines économiques, des manufactures dispersées et des poches de modernité dans le Japon d'avant Meiji, le modèle incarné par 1789 perdait de sa vigueur, et au sein de l'exemple français également, ils allaient rechercher ces traces de modernité.

Ainsi s'explique la focalisation des recherches sur la question de la rente foncière, l'un des problèmes les plus propres à incarner la discussion sur les modes de production. Par leur choix de s'attacher trop exclusivement à cet aspect de l'histoire rurale, les spécialistes japonais de la Révolution française ont été peu sensibles à des questions comme la pression continue qu'exercent les paysans sur les régimes successifs après 1789. Importante seulement à leurs yeux parce que lieu d'apparition de la bourgeoisie, la paysannerie n'existe pas en elle-même comme une actrice majeure du processus révolutionnaire. Les paysans pourraient au contraire être considérés comme acteurs de leur propre libération vis-à-vis du système féodal, et même continuer à influencer le développement particulier du capitalisme français jusqu'au début du $\mathrm{XX}^{\mathrm{e}}$ siècle. A. Ado, dans son ouvrage publié à Moscou en 1971, traduit en français sous le titre Paysans en révolution : terre, pouvoir et jacquerie, 1789-1794, met en évidence la « résistance continue des communautés paysannes à une solution de compromis qui aurait forcé les paysans à rembourser les droits seigneuriaux et féodaux qu'ils payaient avant 1789 ». Ces analyses pressenties par Lefebvre, sont absentes chez Takahashi ou Kawano qui ne parviennent pas à quitter le schéma marxisant, et restent mineures dans une historiographie japonaise des années cinquante pourtant centrée sur le monde rural. 